\title{
Análisis crítico de un artículo: La profilaxis primaria de peritonitis bacteriana espontánea disminuye la aparición de síndrome hepatorrenal y mejora la sobrevida en pacientes cirróticos avanzados
}

\author{
Macarena Roa S $\mathbf{S}^{1, a}$, G abriel Rada G ${ }^{1,2}$. \\ Critically appraised article: \\ Primary prophylaxis of spontaneous \\ bacterial peritonitis delays \\ hepatorenal syndrome \\ and improves survival in cirrhosis \\ Gastroenterology 2007; 133: 818-24. \\ Fernández J, Navasa M, Planas $R$.
}

\begin{abstract}
Background \& Aims: Norfloxacin is highly effective in preventing spontaneous bacterial peritonitis recurrence in cirrhosis, but its role in the primary prevention of this complication is uncertain. Methods: Patients with cirrhosis and low protein ascitic levels $(<15 \mathrm{~g} / \mathrm{L})$ with advanced liver failure (Child-Pugh score $>9$ points with serum bilirubin level $>3 \mathrm{mg} / \mathrm{dL}$ ) or impaired renal function (serum creatinine level $>1.2 \mathrm{mg} / \mathrm{dL}$, blood urea nitrogen level $>25 \mathrm{mg} / \mathrm{dL}$, or serum sodium level $<130 \mathrm{mEq} / \mathrm{L}$ ) were included in a randomized controlled trial aimed at comparing norfloxacin (35 patients) vs placebo (33 patients) in the primary prophylaxis of spontaneous bacterial peritonitis. The main end points of the trial were 3-month and 1-year probability of survival. Secondary end points were 1-year probability of development of spontaneous bacterial peritonitis and hepatorenal syndrome. Results: Norfloxacin administration reduced the 1-year probability of developing spontaneous bacterial peritonitis ( $7 \%$ vs $61 \%, \mathrm{P}<0.001$ ) and hepatorenal syndrome ( $28 \%$ vs $41 \%, \mathrm{P} 0.02$ ), and improved the 3 -month (94\% vs62\%, P 0.003) and the 1 -year ( $60 \%$ vs $48 \%, \mathrm{P} 0.05$ ) probability of survival compared with placebo. Conclusions: Primary prophylaxis with norfloxacin has a great impact in the clinical course of patients with advanced cirrhosis. It reduces the incidence of spontaneous bacterial peritonitis, delays the development of hepatorenal syndrome, and improves survival.
\end{abstract}

\footnotetext{
${ }^{1}$ Unidad de Medicina Basada en Evidencia, Pontificia Universidad Católica de Chile.

${ }^{2}$ Departamento de Medicina Interna, Pontificia Universidad Católica de Chile. Santiago, Chile.

anterno

Correspondencia a: Dr. Gabriel Rada. E mail: gabriel@rada.cl
} 


\section{CONCLUSIÓN DE LOS REVISORES:}

La profilaxis con norfloxacina en pacientes con ascitis por cirrosis avanzada con alto riego de peritonitis bacteriana espontánea (PBE), disminuye la incidencia de PBE, el desarrollo de síndrome hepatorrenal (SHR) y la mortalidad asociada.

\section{PRegunta CĹNICA:}

¿Es útil el uso de antibióticos profilácticos en pacientes cirróticos avanzados con alto riesgo de PBE comparado con placebo, en cuanto a incidencia de PBE, SHR o mortalidad?

\section{ConTeXto:}

La PBE es una infección causada por la translocación bacteriana desde el lumen intestinal a la circulación sistémica y la posterior contaminación del líquido ascítico. Es una complicación frecuente en cirróticos, con una prevalencia que varía entre 10\% y $30 \%$. La recumencia tras un primer episodio de PBE es de $75 \%$ anual y la mortalidad de alrededor de $20 \% 1,2$. Algunos pacientes mueren por shock séptico o una infección incontrolable, mientras que otros fallecen por SHR3.

Si bien la profilaxis secundaria de PBE ha demostrado ser efectiva en disminuir la recurrencia de PBE y la mortalidad ${ }^{4}$, la eficacia de la profilaxis primaria no ha sido adecuadamente estudiada.

Se han identificado distintos factores de riesgo para el desarrollo de $\mathrm{PBE}^{5}$, los que han permitido reconocer a los pacientes en que la prevención parece una medida razonable.

\section{CARACTERÍSTICAS DEL ESTUDIO:}

Tipo de estudio: estudio clínico randomizado, multicéntrico, realizado en 7 hospitales de España entre septiembre de 2000 y junio de 2004. Pacientes: 74 pacientes con cirrosis demostrada por histología, o por la combinación de clínica, laboratorio y ecografía, que tuvieran entre 18 y 80 años y nivel de proteínas en líquido ascítico menor a 1,5 g/dl. Además debían tener alguna manifestación de compromiso renal (creatinina mayor a 1,2 0 BUN mayor a $25 \mathrm{mg} / \mathrm{dl}$ o sodio menor o igual a $130 \mathrm{mEq} / \mathrm{l}$ ) o de falla hepática avanzada (score de Child mayor a 9 puntos, con bilirrubinemia mayor a $3 \mathrm{mg} / \mathrm{dl}$ ). Luego de la randomización se excluyeron 6 pacientes ( 5 por abandono del seguimiento inmediatamente después de la randomización y uno por haber recibido profilaxis anteriormente sin saberlo). Intervención: 35 pacientes recibieron norfloxacino oral 400 mg/día. Comparación: 33 pacientes recibieron placebo del idéntico a la intervención.

La intervención y el placebo se mantuvieron por un año, salvo que el paciente presentara una PBE, SHR, falleciera o se trasplantara en dicho periodo.

VALIDEZ (RIESGO DE SESGO):

\begin{tabular}{|ll|}
\hline 1. Randomizado: & Sí \\
2. Ocultamiento de la secuencia de randomización: & Sí \\
3. Seguimiento: & $93 \%$ \\
4. Análisis con intención de tratar: & Sí, con reparos \\
5. Fueron ciegos a la intervención: & Sí \\
Pacientes: & Sí \\
Tratantes: & Sí \\
Recolectores de datos: & Sí \\
Adjudicadores de eventos: & No claro \\
Analistas de datos: & Sí \\
7. Grupos similares en variables pronósticas conocidas: & No \\
\hline
\end{tabular}




\begin{tabular}{|lcccc|}
\multicolumn{5}{c|}{ Resultados: } \\
\hline $\begin{array}{l}\text { Outcome } \\
\text { (a 3 meses) }\end{array}$ & $\begin{array}{c}\text { Tasa de eventos } \\
\text { grupo intervenido } \\
\%\end{array}$ & $\begin{array}{c}\text { Tasa de eventos } \\
\text { grupo control } \\
\%\end{array}$ & $\begin{array}{c}\text { RR } \\
(\text { IC } 95 \%)\end{array}$ & NNT \\
(IC 95\%)
\end{tabular}

$\mathrm{RR}=$ Riesgo relativo, NNT= Número necesario para tratar, $\mathrm{IC}=$ Intervalo de confianza *Si bien la diferencia no fue significativa a los 3 meses, sí se observó una diferencia estadísticamente significativa en el seguimiento a 12 meses (datos no incluidos en la tabla).

\section{COMENTARIOS:}

Comentarios acerca de la validez (riesgo de sesgo): Se trata de un estudio que cumple con la mayoría de los criterios de validez, al menos parcialmente.

Podría ser cuestionable el hecho de que un paciente fue excluido del estudio luego de la randomización (porque en retrospectiva se determinó que no cumplía los criterios de inclusión), lo cual contradice el principio de intención de tratar. En todo caso, considerando que se trata de un solo paciente, parece improbable que haya introducido sesgo.

Un segundo punto que amenaza la validez es la pérdida de algunos pacientes. Si bien parece poco probable que 5 pacientes hagan una diferencia, podría cambiar la magnitud del resultado e incluso la significación estadística. El que las pérdidas se hayan producido de manera balanceada en ambos grupos disminuye en algo el riesgo de sesgo incorporado por este hecho. Si los pacientes perdidos fueran similares en sus características basales podríamos tener mayor confianza, sin embargo el estudio no presenta esos datos.

Comentarios acerca de los resultados: Este estudio demuestra que la administración de norfloxacino tiene un efecto importante en la profilaxis primaria de pacientes con ascitis y cirrosis avanzada. Se observó un beneficio en outcomes tan relevantes como la incidencia de PBE, el SHR y la mortalidad.

Un aspecto a analizar es la diferencia observada entre los distintos periodos de seguimiento (3 y 12 meses). El efecto observado al tercer mes sobre SHR y mortalidad no se mantuvo al año (datos no incluidos en la tabla), lo cual puede tener dos explicaciones principales. La primera es que efectivamente se pierda el efecto beneficioso de la intervención en forma progresiva; por ejemplo, por la aparición de resistencia al antibiótico o disminución de la adherencia al fármaco. La segunda explicación, es que al disminuir el número de eventos (menos pacientes en seguimiento o menos niesgo basal; ambos factores determinan el poder de un estudio ${ }^{6}$ ) se va perdiendo precisión, lo cual puede producir que el estudio no sea capaz de demostrar una diferencia que realmente existe.

Considerando que a los 12 meses el estimador puntual sigue mostrando una diferencia a favor de la intervención ( $27 \%$ de reducción en la mortalidad y $44 \%$ en la aparición de SHR) y que el efecto sobre la PBE, el desencadenante de los otros outcomes, es claramente significativo (reducción de riesgo relativo de 81\%), nos parece que la segunda explicación es la más probable. 
Dado que se trata de una intervención que podría perder efectividad con el transcurso del tiempo, además de las mencionadas diferencias de significación estadística en los distintos momentos de medición, no se puede asegurar si el efecto se mantendrá más allá de los 12 meses que duró el seguimiento. Así, existe la posibilidad de que al aplicarse por periodos prolongados, los beneficios mencionados disminuyan o no se mantengan. Un periodo de seguimiento más prolongado permitiría, además, observar eventuales efectos adversos no apreciados en el período en cuestión (generación de resistencia a las quinolonas, etc.).

Un punto a destacar es el favorable balance entre beneficio y riesgo. El NNT es de 4 para la mortalidad a los 3 meses, es decir se necesita tratar a 4 pacientes como los de este estudio, por un periodo de 3 meses, para salvar una vida y los efectos adversos son mínimos.

Comentarios acerca de la aplicabilidad: En nuestra opinión, los resultados de este estudio podrían ser aplicados al medio local. En Chile, la cirrosis es una condición frecuente y muchos de los pacientes presentan las características que el estudio define. La intervención es simple, de fácil administración y amplia disponibilidad. Las tasas de deserción fueron bajas, en parte explicadas por la simpleza de la intervención (tomar una pastilla todos los días) y también por el control estricto y cercano de los pacientes (cada 2 meses en el estudio), que constituye un periodo razonable.

El costo de la norfloxacina en dosis de $400 \mathrm{mg} /$ día a permanencia (precio aproximado: $\$ 15.000$ por caja) y del control cada 2 meses, es probablemente menor al de la hospitalización causada por una PBE o SHR y sus eventuales complicaciones. Debe recalcarse que esta intervención estaría ampliamente disponible por cuanto no precisa de un especialista para controlarla. Por otro lado, si bien la norfloxacina fue el antibiótico usado en este estudio, existen alternativas; un estudio randomizado mostró beneficios para el uso de ciprofloxacino en profilaxis primaria de $\mathrm{PBE}^{7}$. Además, el cotrimoxazol y otros antibióticos han sido usados con éxito en profilaxis secundaria de PBE, por lo que razonablemente, al cubrir los mismos agentes, debieran tener efectos positivos sobre la aparición de PBE y sus complicaciones ${ }^{8-13}$.

En conclusión, se trata de una medida eficaz y de costo razonable (especialmente si se utilizan alternativas). Existen algunas dudas acerca de la efectividad a largo plazo de la intervención, lo cual es importante en el momento de informar al paciente acerca de los beneficios y riesgos de esta.

\section{REFERENCIAS}

1. Navasa M, Rimola A, Rodes J. Bacterial infections in liver disease. Semin Liver Dis 1997; 17: 323-33.

2. Wong F, Bernardi M, Balk R, Christman B, Moreau R, Garcia-Tsao G et al. On behalf of the International Ascites Club. Sepsis in cirrhosis: report on the $7^{\text {th }}$ meeting of the International Ascites Club. Gut 2005; 54: 718-25.

3. Folo A, Llovet JM, Navasa M, Planas R, Forns X, Francitorra A et al. Renal impairment after spontaneous bacterial peritonitis in cirrhosis: incidence, clinical course, predictive factors and prognosis. Hepatology 1994; 20: 1495-501.

4. Gines P, Rimola A, Pianas R, Vargas V, Marco F, Almela M et al. Norfloxacin prevents spontaneous bacterial peritonitis recurrence in cirrhosis: results of a double-blind, placebo-controlled trial. Hepatology 1990; 12: 716-24.

5. Liach J, Rimola A, Navasa M, Gines P, Salmeron JM, Gines A. Incidence and predictive factors of first episode of spontaneous bacterial peritonitis in cirrhosis with ascites: relevance of ascitic fluid protein concentration. Hepatology 1992; 16: 724-7.

6. CANDIA R, RADA G. Estudios randomizados interrumpidos precozmente por beneficio: ¿Muy buenos o muy malos? Rev Méd Chile 2006; 134: 1470-5. 
7. Terg R, Fassio E, Guevara M, Cartier M, Longo C, Lucero R et al. Ciprofloxacin in primary prophylaxis of spontaneous bacterial peritonitis: a randomized, placebo-controlled study. J Hepatol 2008; 48: 774-9.

8. Lontos S, Gow PJ, Vaughan RB, Angus PW. Norfloxacin and trimethoprim-sulfamethoxazole therapy have similar efficacy in prevention of spontaneous bacterial peritonitis. J Gastroenterol Hepatol 2008; 23: 252-5.

9. Alvarez RF, Mattos AA, Correa EB, Cotrim HP, Nascimiento TV. Trimethoprim-sulfamethoxazole versus norfloxacin in the prophylaxis of spontaneous bacterial peritonitis in cirrhosis. Arq Gastroenterol 2005; 42: 256-62.

10. Singh N, Gayowski T, Yu VL, Wagener MM. Trimethoprim-sulfamethoxazole for the prevention of spontaneous bacterial peritonitis in cirrhosis: a randomized trial. Ann Intern Med 1995; 122: 5958.

11. Rolachon A, Cordier L, Bacq Y, Nousbaum JB, Franza A, Paris JC et al. Ciprofloxacin and long-term prevention of spontaneous bacterial peritonitis: results of a prospective controlled trial. Hepatology 1995; 22: 1171-4.

12. SoARes-Weiser K, BrEZis M, Leibovici L Antibiotics for spontaneous bacterial peritonitis in cirrhotics. Cochrane Database Syst Rev 2001; (3): CD002232.

13. Fernández J, Ruiz Del Arbol L, Gómez C, Durandez R, Serradilla R, Guarner C et al. Norfloxacin vs ceftriaxone in the prophylaxis of infections in patients with advanced cirrhosis and hemorrhage. Gastroenterology 2006; 131: 1049-56. 